\title{
Prosodically-conditioned factive inferences in Korean: An experimental study*
}

\author{
Sunwoo Jeong \\ Seoul National University
}

\begin{abstract}
Much work has been done to capture the systematic variation in projective behaviors of factive inferences. However, not as much has been said about variation in factivity below the level of projection. This paper presents an experimental study which probes and confirms that Korean showcases patterns of variation in this vein: Factive inferences systematically arise from certain V+CPs, but only under certain prosody, even in unembedded contexts. Characterizing this pattern as prosodically-conditioned factive inferences, the paper proposes a pragmatic analysis of it couched in alternative semantics. The analysis motivates and defends a new interpretive principle which governs how alternatives come into contrast with each other, and re-examines the ontological status of factivity.
\end{abstract}

Keywords: factivity, veridicality, prosody, factive verbs, focus, alternatives, Korean

\section{Introduction}

Semi-factives like realize and know often appear to presuppose the truth of their propositional complements. As demonstrated in (1), this inference generally projects through entailment-canceling operators like negation and antecedents of conditionals, and is intuitively understood to be backgrounded.

(1) a. Lily realized that Mrs. Ramsey was at the party.

b. Lily didn't realize that Mrs. Ramsey was at the party.

c. If Lily realizes that Mrs. Ramsey was at the party, she will be pleased. $\rightsquigarrow$ Mrs. Ramsey was at the party.

However, depending on a variety of factors, the inference may not necessarily project, as exemplified in (2).

(2) If I realize that I had been mistaken, I will confess. $\not \rightarrow$ I had been mistaken.

* I thank the audience at SALT 30 and the 5 anonymous reviewers of the abstract for helpful feedbacks. I also thank Jiyeong Kim for help with preparing the recordings and recruiting participants. This work was supported by the New Faculty Startup Fund from Seoul National University.

(C2020 Jeong 
Much work has been done to capture this systematic variation in projective behaviors of factive inferences, and more generally, other types of 'soft triggers' (Karttunen 1974, Heim 1983, Abusch 2010, Abrusán 2016, Tonhauser 2016, i.a.). However, not as much has been said about variation in factivity below the level of projection. ${ }^{1}$ After all, given the assumption that factivity and (semantic) presupposition are special cases of veridicality and entailment, respectively (roughly, ones that project and are backgrounded), they should not be cancelable or subject to variation in the absence of any entailment-canceling operators. Thus, the veridicality inference (i.e., the entailment about the truth of the complement) is always expected arise in cases like (1a), regardless of whether the verb is construed as a soft trigger or not.

In this paper, we consider whether Korean showcases a pattern that can be adequately characterized as variation in factivity below the level of projection. The starting intuition is as follows: Certain class of Korean verbs such as al- ' $\approx$ know' and gieokha- ' $\approx$ remember', appear to give rise to veridicality inferences that are sensitive to prosody. When the prosodic correlates of focus (their positions henceforth indicated by underlines) fall on the matrix verb, as in (3), the inference about the truth of the complement arises, and is difficult to cancel. However, when the focus falls on any element of the embedded clause, as in (4), the inference doesn't arise (see also Özyıldız (2017b) and Özyıldız (2018) for similar patterns in Turkish).

$$
\begin{aligned}
& \text { Sun-eun }[\text { Byul-i pati-e o-n-jul }]_{p} \text { an-da. } \\
& \text { Sun-NOM [ Byul-NOM party-DAT come-PTCP-C }]_{p} \text { know/believe-DECL } \\
& \approx \text { 'Sun knows that Byul came to the party.' } \rightsquigarrow \text { Byul came to the party. } \\
& \text { Sun-eun }[\text { Byul-i pati-e o-n-jul }]_{p} \text { an-da. } \\
& \text { Sun-NOM [ Byul-NOM party-DAT come-PTCP-C }]_{p} \text { know/believe-DECL } \\
& \approx \text { 'Sun thinks that Byul came to the party.' } \not \rightarrow \text { Byul came to the party. }
\end{aligned}
$$

Furthermore, as long as focus remains on the matrix verb, this inference standardly projects across entailment-canceling operators such as questions, antecedents of conditionals, etc., and is understood be backgrounded, as exemplified in (5). The inference, elicited by appropriate prosody, thus appears to display hallmarks of not just veridicality entailments, but also factive presuppositions.

$$
\begin{aligned}
& \text { Sun-eun [Byul-i pati-e o-n-jul }]_{p} \underline{\text { a-ni? }} \\
& \text { Sun-NOM [Byul-NOM party-DAT come-PTCP-C ] know/believe-Q } \\
& \approx \text { 'Does Sun know that Byul came to the party?' } \rightsquigarrow \text { Byul came to the party. }
\end{aligned}
$$

1 Though see Moulton (2009), Abrusán (2011), Özyıldız (2017a), Lee (2018), and Bondarenko (2019) for discussions of complement-dependent factivity alternation. In this paper, we keep the CP constant as well, and focus on how the same string of words can nevertheless show variation in factive inferences in the absence of entailment-canceling operators, depending crucially on prosody. 
The empirical aim of this paper is to begin establishing a typology of Korean verbs regarding factivity, by quantifying the intuitions outlined in (3)-(4), and comparing them systematically with cases involving other types of Korean verbs. To achieve this, an experimental study involving auditory stimuli is conducted. The resulting data establishes a 3-way contrast between Korean verbs, confirming the presence of a class of verbs whose factivity is systematically dependent on prosody, even in unembedded contexts.

The theoretical aim of this paper is to propose an analysis that can characterize and derive the experimentally observed variations in factive inferences. In developing the account, we engage with the question of what factivity is, and what can function as its 'sources' or 'triggers'. The resulting analysis is couched in alternative semantics (Rooth 1992), and builds on ideas developed in Abusch (2010), Abrusán (2016), and Simons, Beaver, Roberts \& Tonhauser (2017). As in these work, a general pragmatic process which considers relevant alternatives, as constrained by focus, plays an important role. But crucially, in order to move beyond capturing projection variability, we also motivate a new interpretive principle which governs how alternatives come into contrast with each other.

\section{Intuitions and emerging questions}

To facilitate the discussion, let us first establish some terminological shorthands. We will refer to factivity variation in the absence of entailment canceling operators as UC (short for unembedded context) variation, and factivity variation in the presence of entailment canceling operators (i.e., variation at the level of projection), as EC (short for embedded context) variation. So far, we have established that the main focus of this paper is UC variation, and reported on the intuition that Korean verbs such as al- and gieokha- appear to display prosody-sensitive $U C$ variation.

It is difficult to come up with appropriate English glosses/translations for these verbs (i.e., verbs which have been argued to display UC variation). The standard practice is to translate al- as 'know' and gieokha- as 'remember', but as exemplified in (3)-(4), al-and gieokha- do not always convey the truth of their complements in UCs, while the veridicality of their English counterparts in UCs is indisputable. On a first approximation, al- appears to denote an epistemic accessibility relation between an agent and a proposition ${ }^{2}$, and gieokha- a mnemonic accessibility relation. Let us henceforth refer to the former relation as $\mathscr{E}$ and the latter as $\mathscr{M}$, and posit that neither impose any constraints on the relation between the proposition and the actual

2 As noted in Jeong (2020), this appears to be stronger than a mere doxastic relation conveyed by verbs like mit- 'believe'; it is compatible only with beliefs that are based on what the agent considers to be sufficient evidence to constitute knowledge. In light of this, perhaps the most intuitive translation of al- is something like: 'believe to know' or 'believe based on evidence'. See also Özy1ldız (2017a). 
world (i.e., neither encode veridicality).

The intuitions for al- ' $\mathscr{E}$ ' and gieokha- ' $\mathscr{M}$ ', summarized in (3)-(5), contrast with intuitions for other types of Korean verbs such as mit- 'believe', on the one hand, and moreu- 'not know' and ggameok- 'forget' on the other. ${ }^{3}$

First, verbs like mit- 'believe' appear to not give rise to any systematic veridicality inference in UCs or ECs, irrespective of prosody. This is exemplified in (6): Here, we cannot conclude that Byul came to the party, regardless of whether the main verb or an element of the embedded clause bears focus. ${ }^{4}$

$$
\begin{aligned}
& \text { Sun-eun }[\text { Byul-i pati-e o-n-jul }]_{p} \text { mitneun-da. } \\
& \text { Sun-NOM [Byul-NOM party-DAT come-PTCP-C }]_{p} \text { believe-DECL } \\
& \approx \text { 'Sun believes that Byul came to the party.' } \\
& \not \leftarrow \text { Byul came to the party. (across all focus placements) }
\end{aligned}
$$

By contrast, verbs like moreu- 'to not know' and ggameok- 'to forget', appear to always convey veridical inference in UCs, irrespective of prosody. This is exemplified in (7): Here, we can reliably conclude that Byul came to the party, regardless of whether the main verb or an element of the embedded clause bears focus.

$$
\begin{aligned}
& \text { Sun-eun [Byul-i pati-e o-n-jul }]_{p} \text { moreun-da. } \\
& \text { Sun-NOM [ Byul-NOM party-DAT come-PTCP-C ]p not.know-DECL } \\
& \approx \text { 'Sun doesn't know that Byul came to the party.' } \\
& \rightsquigarrow \text { Byul came to the party. (across all focus placements) }
\end{aligned}
$$

Furthermore, the veridicality inference that arises from these verbs appears to standardly project across entailment-canceling operators. For now, we leave the question open as to whether and to what extent the projective behavior of this inference is subject to variation (i.e., what the landscape of EC variation is like).

To recapitulate, Korean attitude verbs appear to be categorized into 3 main types regarding factivity: (i) ones that do not give rise to the inference (e.g., mit- 'to believe'), (ii) ones that do give rise to it: always in UCs and at least most of the time in ECs (e.g., moreu- 'to not know' and ggameok- 'to forget'), and finally, (iii) ones that can systematically elicit it, both in UCs and ECs, but only under certain prosody (e.g., al- ' $\mathscr{E}$ ' and gieokha- ' $\mathscr{M}$ '). Interestingly, verbs identified so far as (ii) vs. (iii) appear to be duals of each other: The former (ii) consists of negative, suppletive counterparts of the latter (iii). This systematic asymmetry between the antonymic duals will play an important role in the analysis to come.

3 In all the examples considered in this section, we fix the complementizer to $j u l$, which, on a first pass, appears to be factively vacuous. See sec. 3.1.2 for more discussion.

4 All glosses follow the Leipzig conventions, with the exception of complementizers, shortened as C. The romanization conventions follow the Revised Romanization of Korean. 
Prosodically-conditioned factive inferences

\section{Experiment}

In order to quantify these intuitions and compare the behaviors of different verbs in a more controlled setting, an experiment was conducted. Its design is inspired by previous experimental work on EC variation (Tonhauser 2016, Djärv \& Bacovcin 2017, Tonhauser, Beaver \& Degen 2018, Mahler, de Marneffe \& Lai 2019), but here the tasks are used to probe UC variation instead (see also: Nairn, Condoravdi \& Karttunen 2006, White \& Rawlins 2018, i.a.).

\subsection{Methods}

\subsubsection{Participants}

71 Native Korean speakers were recruited as participants. The experiment lasted 15-20 minutes. The participants were each remunerated with 5000 won gift cards.

\subsubsection{Stimuli}

Target stimuli were created by crossing 6 attitude verbs (al- ' $\mathscr{E}$ ', moreu- 'not know', gieokha- ' $\mathscr{M}$ ', ggameok- 'forget', alanae- 'discover', mit- 'believe'), 3 complementizers ( $g o, j u l, g e o t)$, and 4 types of prosody.

The 6 verbs were selected based on the discussion in sec. 2. They were predicted to draw out the contrasts of interest revolving around the status of UC variation.

The 3 complementizers (henceforth $\mathrm{Cs}$ ) were chosen by examining which ones combine most naturally and frequently with as many of the verbs listed above as possible. ${ }^{5}$ The resulting combinations are not always fully comparable in naturalness. Based on a variety of lexical semantic properties (which are likely orthogonal to the issue of factivity discussed here), the verbs examined here have slightly different preferences for $\mathrm{Cs}$ and clausal-embedding structures. Nevertheless, to facilitate direct comparisons, all possible crossings of 6 verbs and $3 \mathrm{CP}$ types were included. Among the $3 \mathrm{Cs}$ included in the experiment, we will focus on $g o$ and jul here. As noted in Jeong (2020), geot is associated with nominalization morphology (BogalAllbritten \& Moulton 2018, Lee 2018), such that regardless of the types of verbs they combine with, a sentence with a CP headed by geot is predicted to always give rise to factivity. ${ }^{6}$ By contrast, neither jul nor go appear to function as independent sources of factivity. Consequently, any observed variation in factive inferences involving jul or go most likely stems from the interaction of verb type, prosody, and other contextual factors, rather than the complementizers themselves or the internal structures of CPs.

5 Ones involving geot and jul were further subdivided into cases involving two different internal structures of the CPs: since these elicited comparable results, we treat them as single conditions.

6 This was confirmed by the present experiment. See the link in the appendix for relevant results. 
All sentences emerging from crossing these two factors (verb type * C) were of the format summarized in (8), where MS stands for the matrix clause subject, ES for the embedded clause subject, EV for the embedded verb, $p$ for the embedded proposition (instantiated as 3 items), and MV for the matrix verb.

$$
\text { Structure of auditory stimuli: } \mathrm{MS}\left[C P \underline{\mathrm{ES} \mathrm{EV}}_{p} \mathrm{C}\right] \mathrm{MV} \text {. }
$$

Each of the $54 \mathrm{~V}+\mathrm{CP}$ combinations ( 6 verbs $* 3 \mathrm{Cs} * 3$ items) determined this way was then produced in 4 distinct prosodic patterns representing 4 prosody conditions. The conditions varied systematically on which element obtains prosodic focus. For reasons of space, we concentrate here on 3 conditions: (i) focus on the embedded subject; henceforth the ES condition, (ii) focus on the embedded verb; henceforth the EV condition, and (iii) focus on the matrix verb; henceforth the MV condition.

$$
\begin{aligned}
& \text { a. Byul-eun }[\text { Jin-i hwaga-i-n-jul }]_{C P} \text { an-da. } \\
& \text { Byul-NOM Jin-NOM painter-be-PTCP-C know-DECL. } \\
& \text { b. Byul-eun }[\text { Jin-i hwaga-i-n-jul }]_{C P} \text { an-da. } \\
& \text { Byul-NOM Jin-NOM painter-be-PTCP-C know-DECL. } \\
& \text { c. Byul-eun [Jin-i hwaga-i-n-jul }]_{C P} \text { an-da. } \\
& \text { Byul-NOM Jin-NOM painter-be-PTCP-C know-DECL. }
\end{aligned}
$$

ES

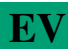

In stress-based accent languages like English, the position of the nuclear pitch accent (NPA) is often argued to be the primary prosodic cue for signaling focus. By comparison, Korean is standardly categorized as a phrasal accent language with no chosen syllables for marking stress. Consequently, focus is often claimed to be signaled by changes in phrasing (beginning of a new 'accentual phrase (AP)' and de-phrasing of the elements following the focused constituent) as well as increase in f0 range, duration, etc. of the word receiving focus (Jun 1996, Jun \& Lee 1998, i.a.). The high-level generalization is that prosodic emphasis, manifested through a variety of acoustic correlates (phrasing, duration, pitch range), provides reliable indications of focus placement. The 4 prosody conditions aimed to systematically incorporate as many putative acoustic correlates of focus as possible. The auditory stimuli were recorded by a K-ToBI trained native speaker in a sound-attenuated room. Posterior acoustic analyses and informal collections of native speaker judgement were conducted to ensure that the stimuli instantiated the intended prosodic conditions.

In the main trials, each target auditory stimulus was associated with a corresponding text stimulus instantiating the complement clause, as in (10).

a. Audio: Byul knows/believes that Jin is a painter.

b. Text: Jin is a painter.

In addition to the target stimuli, 8 control stimuli were created. 4 of them were 
productions of syntactically well-formed (felicitous) Korean sentences, and 4 were productions of syntactically ill-formed (infelicitous) Korean sentences. In the experiment, half of each type (felicitous vs. infelicitous) was associated with text sentences which are either entailed or clearly presupposed by (existential presupposition) the auditory stimuli, and the other half were associated with text sentences which contradicts or does not have any logical relationship with the auditory stimuli.

\subsubsection{Procedure}

Each participant listened to the 36 target stimuli ( 3 out of 4 types of prosody crossed with 6 types of verbs and 2 out of 3 complementizers), and 8 control stimuli, presented in randomized orders (44 trials in total). A given target stimulus was instantiated as 1 of the 3 counterbalanced items representing a variety of embedded propositional contents. In a given trial, the speaker was identified alongside the recording. After listening to each recording, two questions were posed.

The speaker X says: [AUdIOSTIMULUS]

Q1: How natural does the recording sound?

Q2: Given what you heard, how likely are you to conclude as follows?

The speaker $\mathrm{X}$ thinks: [TEXTSTIMULUS $]_{p}$

Q1 inquired about the acceptability of the target stimuli. The responses to this will henceforth be referred to as naturalness ratings. This task was included primarily to confirm that all possible crossings between the verbs and the complementizers tested in the experiment sound acceptable. As we will see in more detail in sec. 3.2.2, not all pairings were in fact judged to be felicitous, and these gaps will turn out to have some bearing on the analysis of the main results on factive inferences (sec. 4.4).

The main task of interest is Q2. In the target trials, Q2 inquired about the likelihood that the speaker of the utterance presumes the truth of the complement. The question is akin to the ones adopted in Tonhauser (2016), and functioned as a probe for tracking whether veridicality inferences arose or not. The responses to this will henceforth be referred to as veridicality ratings. In the control trials, Q2 invited the participants to rate the validity of the text inferences. Both Q1 and Q2 elicited ratings in discrete, 7-point Likert scales.

\subsection{Results}

\subsubsection{Methods for analysis}

To facilitate the interpretation and the discussion, the resulting data were subdivided into 2 sets depending on $\mathrm{C}$ (jul set and $g o$ set). To each set, mixed effects ordinal 
regression models ( $\mathrm{M} 1_{j u l / g o}, \mathrm{M} 2_{j u l / g o}$ ) were fitted: The main dependent variable was the naturalness rating $\left(\mathrm{M} 1_{j u l / g o}\right)$ or the veridicality rating $\left(\mathrm{M} 2_{j u l / g o}\right) .^{7}$ The independent variables were verb type and prosody, with interactions between the two. Random intercepts were posited for items and participants.

\subsubsection{Naturalness (Q1)}

While responses to Q1 aren't our main data of interest, two observations are worth mentioning. First, pairings between $j u l$-CPs and the 6 verbs were all judged to be reasonably acceptable, eliciting significantly higher naturalness ratings than infelicitous controls. ${ }^{8}$ Second, pairings between go-CPs and moreu- 'not know' and ggameok- 'forget' were judged to be categorically unacceptable, eliciting low naturalness ratings which were not significantly different from infelicitous controls. ${ }^{9}$ Put briefly, participants' responses to Q1 suggest that jul can combine with any of the 6 verbs investigated here, whereas go cannot combine with certain negative attitude verbs.

\subsubsection{Veridicality $(\mathrm{Q} 2)$}

Let us now turn to the data of interest. The core prediction for the veridicality ratings was that 3 distinct factivity patterns (outlined in sec. 2) would emerge for Korean verbs, both in cases of $j u l$ and $g o$.

With regards to cases involving $j u l$, the experimental results confirm this prediction. Fig. 1 summarizes this state of affairs. In fig. 1, the x-axes and the bar colors represent the 3 prosody conditions, and the $y$-axes represent mean veridicality ratings (participants' answers to Q2). Each columns of the panels represent the 6 types of verbs tested, and each rows of the panels represent the 2 Cs of interest. ${ }^{10}$ Finally, the yellow dotted lines superimposed on the bars represent mean validity ratings for entailment controls, and the grey dotted lines represent mean validity ratings for no-entailment controls. These are intended to function as reference levels for

7 See the link in the appendix for full model summaries and visualizations of the naturalness ratings. 8 At the same time, some finer differences could be observed depending on the verb type: jul-CPs elicited the highest naturalness ratings when paired with al- ' $\mathscr{E}$ ' and moreu- 'not know', reflecting the native speaker intuition that $j u l$ combines most productively with epistemic verbs.

9 As alluded to in sec. 3.1.2, the reason for this is likely orthogonal to the issue of factivity discussed here. Roughly put, go has a quotative flavor, and the infelicity of moreu- 'not know' and ggameok'forget' combined with go likely stems from the fact that it is difficult (if not impossible) to conceive of quoting someone on a proposition they don't know or remember.

10 For ease of interpretation, the veridicality ratings for pairings between go and moreu- 'not know' / ggameok- 'forget' have been omitted from fig. 1. This is because as noted in sec. 3.2.2, these were judged to be categorically unacceptable by native speakers. Full results can be found in the appendix. 

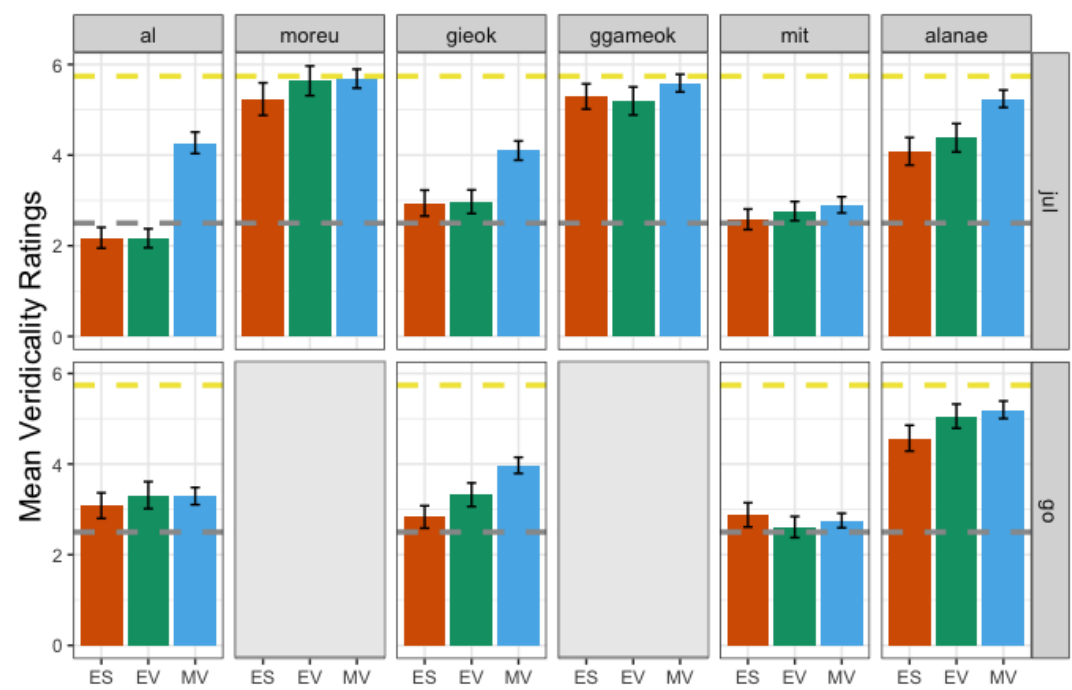

Focus on Embedded Subject:

Focus on Embedded Verb:

Focus on Main Verb:

Figure 1 Veridicality ratings. Means and standard errors.

evaluating the veridicality ratings.

From the first row of panels (cases involving $j u l$ ), 3 main patterns can be identified. First, the verb mit- 'believe' elicits low veridicality ratings overall, irrespective of prosody. In particular, their veridicality ratings are significantly lower than those elicited by verbs such as moreu- 'not know', and closely track the validity ratings elicited from contradiction and other no-entailment controls. All of these suggest that as predicted, mit-type verbs do not give rise to any veridicality entailment. In the model (M2), this was captured by a significant main effect of verb type (e.g., comparison with moreu-: $\beta=3.07, S E=0.47, z=6.43, p<.001$ ) and no main effect of prosody (when mit- is set as the reference level).

Second, the verbs moreu- 'not know' and ggameok- 'forget', henceforth referred to as moreu-type verbs, elicit high veridicality ratings overall, irrespective of prosody. In particular, their veridicality ratings are significantly higher than those elicited by mit- 'believe', as well as those elicited by al- ' $\mathscr{E}$ ', and gieok- ' $\mathscr{M}$ ', and closely track the validity ratings elicited from entailment controls. All of these suggest that as predicted, moreu-type verbs reliably give rise to veridicality entailments across all prosodic conditions. In the model (M2), this was again captured by a significant main effect of verb type (e.g., comparison with al-: $\beta=-3.67, S E=0.47, z=-7.69$, $p<.001$ ) and no main effect of prosody (when moreu- or ggameok- is set as the reference level).

Finally, the verbs al- ' $\mathscr{E}$ ' and gieokha- ' $\mathscr{M}$ ', henceforth referred to as al-type 
verbs, confirm the presence of prosody-sensitive factivity variation predicted in sec. 1: these verbs elicit significantly higher veridicality ratings in MV condition than in the other two prosody conditions (ES, EV). In the model (M2), this was captured by a significant main effect of prosody (e.g., ES compared with MV: $\beta=2.35, S E=0.40, z=5.78, p<.001$ ) for al-type verbs, as well as a significant interaction between verb type and prosody, more globally. Still, even in the MV condition, the veridicality ratings of al- ' $\mathscr{E}$ ' and gieokha- ' $\mathscr{M}$ ' were significantly lower than those associated with moreu-type verbs.

Let us now turn to data involving go, which is summarized in the second row of fig. 1. Here, contra our expectations, al-type verbs do not appear to derive prosodysensitive veridical inferences anymore, and seem to behave more like mit-type verbs. Confirming this, the interaction between verb type and prosody was no longer significant in the model.

\subsection{Variation in EC contexts}

In a smaller scale, another pilot experiment was conducted with an analogous main task, but with target sentences embedded under entailment-canceling operators. Though more data are needed, broadly, the preliminary results confirm that the relevant veridicality inference projects, both in the case of moreu-type verbs and in the case of al-type verbs (the latter, again only in the MV prosody condition). Based on this, we provisionally conclude that the relevant inference is not only veridical, but also factive in nature (see sec. 5 for more discussion).

\section{Analysis}

The experimental results establish a 3-way contrast between Korean verbs regarding factivity, and confirm the presence of prosody-sensitive UC variation for al-type verbs. In this section, we propose an analysis that can capture these results.

Can analyses which aim to capture projection variability (i.e., EC variation) be straightforwardly applied to the emerging UC variation data? The short answer appears to be no. For instance, analyses which explicate the variation in terms of local vs. global accommodation (Heim 1983, van der Sandt 1992, i.a.) can't be applied, as the evaluating contexts are always 'local' in cases of UC variation. Similarly, analyses which reduce the presuppositions of 'soft triggers', including semi-factives, to entailments (in UCs) or scalar implicatures (in ECs; Chemla 2009, Romoli 2014) can't be applied here either, as these presume a particular alternative structure where at least the veridicality entailment of the attitude verbs is lexically specified from the outset.

Another line of work explicates EC variation by positing a general pragmatic 
process where the disjunction of salient pragmatic alternatives are evaluated, triggering a particular inference (Abusch 2010, Abrusán 2016, Simons et al. 2017). While this line of approach is also not straightforwardly applicable to the current UC data without further ado, the analysis developed in this paper inherits and extends its main ideas, instantiated as (12c). Including this piece, the present alternatives-based analysis of the Korean UC variation has four main components, summarized in (12).

a. Lexical asymmetry Certain attitude verbs in Korean lexically encode veridicality/factivity, whereas their salient counterparts don't

b. Focus and alternatives Focus, indicated by prosody, systematically constrains pragmatic alternatives

c. The disjunctive inference It is standardly presupposed that the disjunction of these pragmatic alternatives is true

d. Unique at-issue contrast Alternatives that enter into this disjunctive inference only allow for a single dimension of semantic contrast

Let us now go over each component in more detail.

\subsection{Lexical asymmetry}

The experimental results outlined in sec. 3.2 confirm the presence of an interesting asymmetry: positive attitude verbs like al- ' $\mathscr{E}$ ' and gieokha- ' $\mathscr{M}$ ' generate factive inferences only under certain prosody in UCs, but their negative counterparts moreu'not know' and ggameok- 'forget' always generate factive inferences in UCs. On the basis of this, we posit that moreu-type negative verbs lexically encode factivity (or at the very least, veridicality), whereas al-type positive verbs don't (12a). How exactly factivity is encoded in negative verbs would depend partly on how projection variability (EC variation) is accounted for, assuming that such variability exists (e.g., Is the presuppositional status encoded semantically as a definedness condition, or is the inference encoded more like standard entailments, with projection being explicated by independent, pragmatic mechanisms?). For now (since we are focusing solely on UC variation), it suffices to adopt a simple conjunctive analysis (Stalnaker 1977), and posit that however they may arise, sentences containing moreu-type verbs semantically/conventionally give rise to two entailments, whereas those containing al-type verbs only give rise to one, as summarized in (13) vs. (15).

$$
\begin{array}{ll}
\text { a. } & \llbracket x p \text { al- } \rrbracket=\mathscr{E}_{w}(x, p) \quad(14) \quad \llbracket x p \text { mit }-\rrbracket=\mathscr{D}_{w}(x, p) \\
\text { b. } & \llbracket x p \text { gieok }-\rrbracket=\mathscr{M}_{w}(x, p) \\
\text { a. } & \llbracket x p \text { moreu- } \rrbracket=\neg \mathscr{E}_{w}(x, p) \wedge p(x) \\
\text { b. } & \llbracket x p \text { ggameok }-\rrbracket=\neg \mathscr{M}_{w}(x, p) \wedge p(x)
\end{array}
$$


In this sense, at the semantic level, we assume that verbs like $a l$ - ' $\mathscr{E}$ ' and gieokha- ' $\mathscr{M}$ ' are analogous to categorically non-factive, non-veridical verbs like mit- 'believe'. The latter of course, are predicted to not encode any veridicality, as summarized in (14). The verb mit- for instance, is predicted to only encode that a doxastic accessibility relation $\mathscr{D}$ holds between an agent and a proposition.

Assuming that the lexical entries we posit for the relevant attitude verbs are as in (13)-(15), ${ }^{11}$ our main research question can now be reformulated as follows: How do only al-type verbs come to obtain factive inferences when the MV bears prosodic markings of focus? Put differently, how are they different from mit-type verbs?

\subsection{Prosody, information structure, and the disjunctive inference: VALT}

Why and how does prosody come to affect veridicality/factivity, specifically that of al-type verbs? We begin answering this question by adopting the view from focus/alternative semantics (Rooth 1992) that prosodic emphasis marks focus, and that focus generates alternatives, thereby signaling the information structure of a given utterance (12b). Under this view, a focus bearing expression such as $\operatorname{Jin}_{F}$ is associated with the ordinary semantic value like the one in (16a), and the focus semantic value like the one in (16b), which is a set containing the former and its domain alternatives.

$$
\begin{array}{ll}
\text { a. } & \llbracket \mathrm{Jin} \rrbracket^{o}=\mathrm{JIN} \\
\text { b. } & \llbracket \mathrm{Jin} \rrbracket^{f}=\left\{x \mid x \in D_{e}\right\}=\{\mathrm{JIN}, \text { IRENE, WENDY, } \ldots\}
\end{array}
$$

In the experiment, the 3 prosody conditions instantiated different focus placements. Consequently, they are predicted to generate systematically different focus alternatives. Assuming that these subsequently undergo point-wise functional application, the alternatives generated by the sentence (17) in ES vs. MV condition would be as in (17a) vs. (17b), respectively. (Note that the alternatives in the MV focus condition (17b) vary in relation $R$ denoted by the MV, and therefore include an alternative containing moreu- (15a), among others.)

$$
\begin{aligned}
& \text { Byul-eun }[\text { Jin-i hwaga-i-n-jul }]_{C P} \text { an-da. } \\
& \text { Byul-NOM Jin-NOM painter-be-PTCP-C know/believe-DECL. }
\end{aligned}
$$

a. $\llbracket(17)_{\mathbf{E S}} \rrbracket^{f}=\{\text { Jin is a painter, Irene is a painter, ... }\}^{12}$

11 One may ask why there exists a lexical asymmetry between al-type vs. moreu-type verbs in the first place. Perhaps it is because talking about someone not having an epistemic/mnemonic relation to a proposition has utility primarily when that proposition is true, i.e., matters. More investigation is needed however, to further elucidate this issue.

12 Alternatively, if point-wise functional application proceeds beyond the embedded clause, the resulting focus alternatives would be: $\left\{\mathscr{E}_{w}\right.$ (Byul, Jin is a painter $), \mathscr{E}_{w}$ (Byul, Irene is a painter $\left.), \ldots\right\}$. 
Prosodically-conditioned factive inferences

b. $\llbracket(17){ }_{\mathbf{M V}} \rrbracket^{f}=\left\{\mathscr{E}_{w}(\right.$ Byul, Jin is a painter $), \neg \mathscr{E}_{w}($ Byul, Jin is a painter $)$ $\wedge($ Jin is a painter $), \ldots\}$

With this general prosody-alternatives connection in place, we additionally posit that focus alternatives like (17a) and (17b) systematically structure context-sensitive pragmatic alternatives of a given utterance. To capture this, we follow Simons et al. (2017) in assuming that a given utterance $\varphi$ is associated with a salient pragmatic alternative set $\mathrm{ALT}_{\varphi}$, which is a contextually constrained, non-empty, non-singleton subset of focus alternatives $\llbracket \varphi \rrbracket^{f, g}$ including $\varphi$ itself.

In determining the appropriate $\operatorname{ALT}_{\varphi}$ sets for the cases at hand, we make one final assumption: By default, moreu- 'not know' functions as a salient alternative to $a l-' \mathscr{E}$ ' and figures in the $\mathrm{ALT}_{\varphi}$ set of the latter, and vice versa. Likewise for the pair ggameok- 'forget' and gieokha- ' $\mathscr{M}$ '. This is based on the native speaker intuition that each pair is often considered to be a kind of dual, which 'go together' (this intuition is more pronounced for $a l$ - and moreu-). Furthermore, presuming such a default alternative facilitates the reconstruction of what seems to be a common issue-under-discussion signaled by the MV prosody, namely, whether a particular attitudinal relation holds or not between an agent and a proposition.

The $\operatorname{ALT}_{\varphi}$ set functions as inputs/arguments to a variety of alternative-sensitive pragmatic procedures. Following the ideas propounded in Abusch (2002), Abusch (2010), Abrusán (2016), and Simons et al. (2017), we claim that one such process is the disjunctive inference (12c). Specifically, listeners reason pragmatically that the disjunction of the elements in $\mathrm{ALT}_{\varphi}$, i.e., $\mathrm{VALT}_{\varphi}$, is presupposed (i.e., entailed by the context; see also theories of Givenness in Schwarzschild (1999), Büring (2016), and Rochemont (2016)). This assumption can capture, among other things, defeasible existential presuppositions of focused expressions, and has been productively used to capture projection variability (i.e., EC variation).

There exists a number of ways to motivate this putative default pragmatic mechanism. For instance, alternatives can be construed as identifying what is at-issue in a discourse, and reflecting the conversational goal of determining which of the alternatives actively being considered is true. Considering this, the inference that is guaranteed to arise regardless of which alternative ends up being chosen is likely to function as backgrounded, established information. Insights along this vein is operationalized by Simons et al. (2017) via the notion of CQ (current question), which is equivalent to the notion of QUD (Question Under discussion; Roberts 1996): $\mathrm{ALT}_{\varphi}$ is essentially CQ, and whatever is already entailed by the CQ is not at-issue and thus projects, where CQ is taken to entail the disjunction of its cells (i.e., its possible answers). Regardless of how exactly we motivate the process, what is important for the current analysis is that we presume some general process which evaluates all the contextually salient alternatives (as constrained by focus) holistically, and derives a 
certain inference about what is (indicated to be) already established.

\subsection{Towards correct predictions}

With the ingredients established so far, we can already start deriving a few correct predictions. In particular, the lexical asymmetry posited in sec. 4.1 plays a pivotal role in predicting that al-type verbs don't generate factive inference under ES/EV focus, whereas moreu-type verbs do. Consider for instance, cases involving alvs. moreu- with ES focus. Since al-does not lexically encode factivity (13a), the ordinary semantic value of the al-case (18a), which is unaffected by prosody, doesn't give rise to any factive entailment. Furthermore, since the ALT set, as structured by focus alternatives, would be as in (18b), the only presuppositional inference that is predicted to emerge from the disjunctive process VALT is the inference that someone is a painter. ${ }^{13}$ In short, we correctly predict that $\vee A L T$ will not contribute any independent factive inference in cases involving ES focus (and by extension, $\mathrm{EV}$ focus, applying the same line of reasoning) for al-type verbs.

Byul-eun $[\underline{\mathrm{Jin}}-\mathrm{i} \text { hwaga-i-n-jul }]_{C P}$ an-da. Byul-NOM Jin-NOM painter-be-PTCP-C know/believe-DECL

a. $\llbracket(18) \rrbracket^{o}=\mathscr{E}_{w}($ Byul, Jin is a painter $)$

b. $\quad \operatorname{ALT}_{(18)}=\{$ Jin is a painter, Irene is a painter, ... $\}$

c. $\operatorname{VALT}_{(18)} \rightsquigarrow$ Someone is a painter.

Byul-eun [Jin-i hwaga-i-n-jul $]_{C P}$ moreun-da. Byul-NOM Jin-NOM painter-be-PTCP-C not.know-DECL

a. $\llbracket(19) \rrbracket^{o}=\neg \mathscr{E}_{w}$ (Byul, Jin is a painter $) \wedge($ Jin is a painter $)$

b. $\operatorname{ALT}_{(19)}=\{$ Jin is a painter, Irene is a painter, ... $\}$

When the embedded subject is focused in the moreu- case, we derive an analogous ALT set. We therefore predict that again, the disjunctive process VALT will only give rise to the presupposition that someone is a painter. Crucially however, moreu- lexically encodes factivity (15a). Therefore, the ordinary semantic value of this sentence (19a), which is unaffected by prosody, is predicted to give rise to a factive inference (that Jin is a painter) anyway, under all 3 prosody conditions.

So far, the disjunctive process has taken a back seat in generating the correct predictions for ES/EV cases. Based on the works on EC variation, we expect it to play a more central role in deriving the factive inference of al-type verbs under the appropriate prosody. However, the disjunctive process and the lexical entries by

13 If we instead posit the alternatives entertained in footnote 12, the predicted inference would be that Byul knows that someone is a painter. Native speaker intuitions confirm that both types of inferences arise robustly in standard contexts from ES focus cases like (18)-(19). 
themselves still cannot explain why al-type verbs come to obtain factive inferences under MV focus. For instance, when $a l$ - is focused (17b), we predict that the ALT would be structured as in (20a): The set contains $\varphi$ itself, as well as the default alternative involving moreu- ( $p$ stands for the embedded proposition).

$$
\begin{aligned}
& \text { Byul-eun }[\text { Jin-i hwaga-i-n-jul }]_{p} \quad \underline{\text { an-da. }} \\
& \text { Byul-NOM Jin-NOM painter-be-PTCP-C know/believe-DECL. }
\end{aligned}
$$

a. $\operatorname{ALT}_{(20)}=\left\{\mathscr{E}_{w}(\right.$ Byul, $p), \neg \mathscr{E}_{w}($ Byul, $\left.p) \wedge p\right\}$

b. $\operatorname{VALT}_{(20)}=\left(\mathscr{E}_{w}(\right.$ Byul, $\left.p)\right) \vee\left(\neg \mathscr{E}_{w}(\right.$ Byul, $\left.p) \wedge p\right)$

If we are to maintain the lexical asymmetries between al- and moreu- (13a) vs. (15a), which were crucial to generating the correct predictions in (18)-(19), then $p$ cannot follow from the disjunctive process VALT, as captured in (20b). It thus appears that the disjunctive process and alternative semantics, which have been successfully used to capture EC variation, are not sufficient to capture UC variation, i.e., variation below the level of projection. We need something more.

\subsection{An interpretive principle: A single at-issue contrast}

We argue that what is crucially needed is an independently motivated interpretive principle. The interpretative principle is christened as 'Uni-dimensional Heterogeneity of Alternatives (henceforth, UHA)', and is defined as in (21).

(21) UHA: Elements of a discourse salient set of alternatives $\operatorname{ALT}_{\varphi}$ that enter into the disjunctive pragmatic inference $\vee \mathrm{ALT}_{\varphi}$ only allows for a single dimension of semantic contrast.

While the formulation of UHA remains somewhat general at the moment, the implementation of UHA in the domain of attitude predicates can be elaborated further as follows. Attitude verbs can in principle encode information along two semantic axes: (A) relation between proposition $p$ and agent $x$ 's mental state (the attitudinal dimension), and (B) relation between proposition $p$ and the actual world (the veridical dimension). Lexically, moreu-type verbs specify both: (A) $\neg \mathscr{E}_{w}(x, p)$ and (B) $w \in p$, whereas al-type verbs only specify one: (A) $\mathscr{E}_{w}(x, p)$. Crucially, whenever both types of verbs co-exist in $\mathrm{ALT}_{\varphi}$, UHA enforces them to contrast with each other only along one of the two axes: (A) or (B).

With this in mind, let us return to the case of interest: (20). The analysis predicts that when $\mathrm{ALT}_{\varphi}$ evokes a paradigmatic contrast between a clause containing a nonveridical verb like $a l$ - and a clause containing a veridical verb like moreu-, al- needs to be enriched, and in effect be interpreted as $\mathscr{E}_{w}(x, p) \wedge p(x)$ in order to observe UHA. This is because $a l$ - and moreu-already contrasts along the (A) axis, and (B) 
is already fixed for moreu- (whereas it is underspecified, i.e., not encoded one way or the other, for $a l$-). We thus obtain the correct inference that $p$ for (20), because: $\left(\mathscr{E}_{w}(\right.$ Byul,$\left.p) \wedge p(w)\right) \vee\left(\neg \mathscr{E}_{w}(\right.$ Byul,$\left.p) \wedge p(w)\right) \rightsquigarrow p(w)$.

The addition of UHA preserves all the interim predictions we derived in sec. 4.3. For instance, al-cases under ES/EV focus (18) are still predicted to not derive any factive inference. This is because the ALT sets here (18b) observe UHA from the outset: the elements in the set vary only along a single semantic dimension, namely, the identity of the agent, and no further interpretive enrichment is needed.

In a similar vein, we can now generate the additional prediction that no factive inference would arise for mit-type verbs, even under MV focus. Unlike al- and gieokha-, which appear to be associated with default antonymic alternatives that are lexically factive, mit- 'believe' appears to not be associated with a salient factive alternative. While a salient default alternative does not come as readily to mind for mit-, some likely candidates are uishimha- 'doubt' or an mit- 'not believe'. ${ }^{14}$ Crucially, native speaker intuitions confirm that neither uishimha- nor an mit-give rise to veridicality entailments. Assuming that mit- standardly enters into an at-issue contrast with these other non-factive alternatives when focused, we predict that it can observe UHA without having to undergo any factive enrichment: the elements in the ALT set would vary only along the (A) dimension from the outset.

Importantly, we claim that UHA is not a mere stipulation, but is motivated by general pragmatic considerations. An idea along this vein was adumbrated in Stalnaker (1977), where he proffers a pragmatic account of the factive presupposition of know. Roughly, he claims that if the speaker were to use 'know' to assert both (i) that a certain attitudinal relation holds between an agent and a proposition, and (ii) that the proposition is true (i.e., if both points were at-issue and not taken for granted), then she would be 'saying in one breath something that could be challenged in two different ways'. As Abusch (2010) notes, Stalnaker (1977)'s account runs into problems in its bare form. With certain refinements however, its key intuition may be preserved under new forms. The UHA proposed here can be construed as a particular way of refining Stalnaker's central insight, by incorporating a necessary structural background via the notion of $\operatorname{ALT}_{\varphi}$. In short, UHA is motivated by the communicative desire to convey a uniquely identifiable at-issue contrast (a single main point, if you will), and is sensitive to information structures of specific utterances in its application. While not actively pursued here, UHA can be recast under a CQ-based analysis: as CQ represents the current discourse topic in a question form, it makes sense to assume that CQ would require its answer cells to be structured in a principled way (e.g., share the same presupposition).

The current analysis has the added advantage of capturing the unexpected com-

14 This involves short negation, which is a type of construction that is not made available for verbs like al- and gieokha-, perhaps because they have suppletive counterparts instead. 
plexities in the experimental results (sec. 3.2.3). In particular, recall that al-type verbs no longer generated factive inferences under MV focus when combined with the complementizer $g o$. This can be captured straightforwardly by the fact that the pairings between moreu-type verbs and go were judged to be unacceptable, due to an orthogonal semantic clash between the two (sec. 3.2.2). This means that the negative suppletive counterparts of $a$-type verbs, which by default figure as salient factive alternatives in $j u l$ cases, can no longer function as viable discourse alternatives in $g o$ cases. Consequently, we predict that in cases involving go, focused $a l$-type verbs are much more likely to contrast with other non-factive alternatives, and UHA can in turn be observed without factive enrichment of the main verb. ${ }^{15}$

Having gone over the analysis, let us return to the questions we started out with. Can we characterize inferences triggered by al-type verbs under MV prosody as factive? If factivity is construed as a special kind of entailment that arises from from lexical, conventional specifications of certain words, then al-type verbs are definitely not factive verbs (i.e., presupposition triggers) in the traditional sense: They do not themselves semantically/conventionally encode factivity. Unlike mit-type verbs however, they evoke lexically factive alternatives by default. These, interacting with general interpretive processes/principles such as VALT and UHA, preferentially endow al-type verbs with a systematic inferential channel of deriving factivity. Considering this, we may provisionally conclude as follows: If 'factivity' can refer to any difficult-to-cancel, backgrounded, projective veridicality inference (however they may arise), then al-type verbs can be said to generate properly factive inferences under certain prosody. The 'source' or 'trigger' of factivity in this case boils down to the landscape of alternative expressions.

\section{Discussion, conclusion, and remaining questions}

In this study, we established that Korean verbs can be divided into 3 main types depending on their factivity, and demonstrated that one of them displays prosodysensitive factivity variation in unembedded contexts (UC). To capture this empirical pattern, we proposed that interpretations of verbs that are presuppositionally underspecified can nevertheless obtain factive interpretations when their contrasting factive alternatives are activated. A combination of a lexical asymmetry, a general disjunctive inference $V A L T$, and a principle governing how elements of ALT are

15 Essentially the same line of explanation can be used to capture the observed gradience in veridicality ratings of $a l$-type verbs $+j u l$ under MV prosody (their veridicality ratings were high, but still significantly lower than those of moreu-type verbs). If, in particular contexts, $a l$ - is able to enter into an at-issue contrast with another non-factive alternative (instead of the default factive alternative moreu-), then we would predict that UHA can be observed without factive enrichment in al-cases with MV prosody. More empirical investigation is needed however (perhaps ones incorporating explicit prior contexts), to clarify this issue. See sec. 5 and Jeong (In prep) for more discussion. 
structured (UHA), all of which have intuitive and independent motivations, fully capture the systematic, prosody-sensitive factivity variation in Korean.

On the surface, the prosody sensitivity of UC variation documented in this study is reminiscent of the prosody sensitivity of English EC variation noted in Beaver (2010) and Tonhauser (2016), i.a.: In both cases, narrow focus on an element of the $\mathrm{CP}$ is observed to discourage factive inference from arising (or projecting), whereas focus on the main verb (MV) is observed to facilitate the derivation of the inference. However, the mechanism via which the UC variation is captured in the current analysis is a bit different from the one posited for the EC variation: What matters for theories of $\mathrm{EC}$ variation is that the complement clause be not-at-issue (as only not-at-issue contents are predicted to project; e.g., Simons et al. 2017). Regarding UC variation investigated here however, what matters is that the main verb be at-issue: This is how, in a given context, a non-factive verb can enter into a paradigmatic contrast with a salient factive alternative and obtain a factive interpretation, in order to maintain a single at-issue contrast. However, in terms of the big picture, the two explanatory mechanisms share a common core in that information structure plays a central role in determining the presence of factive inference. Further work is needed to clarify if and how other informational structural notions such as givenness (Schwarzschild 1999, Wagner 2012, Büring 2016, Rochemont 2016) relate to phenomena of 'gradient' factivity (White 2019, i.a.).

Many other questions still remain, and we conclude by highlighting just a few of them. The first concerns the exact scope and implementation of UHA, as well as its cross-linguistic implications (e.g., Can there be repairs in which the attitudinal dimension, and not the veridical dimension, is homogenized? (Wataru Uegaki, p.c.); Do we predict all languages with prosodic UC variation to involve the activation of UHA? (Tanya Bondarenko, Deniz Özyıldız, p.c.)). The second involves the status of 'default' salient alternatives of certain expressions and the role of prior contexts (Roger Schwarzschild, p.c.): How is the default preference specified/represented, and how does context comes into play in determining or overriding it? The third involves the landscape of EC (projection) variation in languages like Korean, which is characterized by systematic UC variation. The last issue involves the veridicality behaviors of Korean verbs in the domain of interrogative complements (Spector \& Egré 2015, Theiler, Roelofsen \& Aloni 2018, Steinert-Threlkeld 2020, Roelofsen \& Uegaki 2020). On a first approximation, al-type verbs appear to display typologically unexpected patterns (they are selectively veridical with $p$, but obligatorily veridical with $Q$ complements) worth investigating further.

\section{A Experiment}

Experiment, data, and codes can be found at: https://github.com/sunwooj/veridicality. 
Prosodically-conditioned factive inferences

\section{References}

Abrusán, Márta. 2011. Predicting the presuppositions of soft triggers. Linguistics and Philosophy 34(6). 491-535. doi:10.1007/s10988-012-9108-y.

Abrusán, Márta. 2016. Presupposition cancellation: explaining the 'softhard' trigger distinction. Natural Language Semantics 24(2). 165-202. doi:10.1007/s11050-016-9122-7.

Abusch, Dorit. 2002. Lexical alternatives as a source of pragmatic presuppositions. Semantics and Linguistic Theory (SALT) 12. 1-19. doi:10.3765/salt.v12i0.2867.

Abusch, Dorit. 2010. Presupposition triggering from alternatives. Journal of Semantics 27(1). 37-80. doi:10.1093/jos/ffp009.

Beaver, David. 2010. Have you noticed that your belly button lint colour is related to the colour of your clothing? In R Bauerle, U Reyle \& T. E. Zimmermann (eds.), Presuppositions and discourse: Essays offered to Hans Kamp, 65-99. Oxford, UK: Elsevier. doi:10.1163/9789004253162_004.

Bogal-Allbritten, Elizabeth \& Keir Moulton. 2018. Nominalized clauses and reference to propositional content. Sinn und Bedeutung (SuB) 21(1). 215-232.

Bondarenko, Tanya. 2019. Factivity alternation due to semantic composition: think and remember in Barguzin Buryat. Generals paper, MIT.

Büring, Daniel. 2016. Intonation and Meaning. Oxford: Oxford University Press.

Chemla, Emmanuel. 2009. Presuppositions of quantified sentences: Experimental data. Natural Language Semantics 17(4). 299-340. doi:10.1007/s11050-0099043-9.

Djärv, Kajsa \& Hezekiah Akiva Bacovcin. 2017. Prosodic effects on factive presupposition projection. Semantics and Linguistic Theory (SALT) 27. 116-133. doi:10.3765/salt.v27i0.4134.

Heim, Irene. 1983. On the projection problem for presuppositions. In M Barlow, D. P. Flickinger \& M. T. Wescoat (eds.), The 2nd West Coast Conference on Formal Linguistics (WCCFL 2), 114-125. Stanford, CA: Stanford Linguistics Association.

Jeong, Sunwoo. 2020. The effect of prosody on veridicality inferences in Korean. In New Frontiers in Artificial Intelligence (LNCS, volume 12331), Yokohama, Japan: Springer International Publishing. doi:10.1007/978-3-030-58790-1_9.

Jeong, Sunwoo. In prep. Melodies of truth: Deriving factive inferences from alternatives. Manuscript.

Jun, Sun-Ah. 1996. The phonetics and phonology of Korean prosody: Intonational phonology and prosodic structure. Taylor \& Francis. doi:10.4324/9780429454943.

Jun, Sun-Ah \& Hyuck-Joon Lee. 1998. Phonetic and phonological markers of contrastive focus in Korean. In Robert H. Mannell \& Jordi Robert-Ribes (eds.), 
The 5th International Conference on Spoken Language Processing (ICSLP 5), 1295-1298. Canberra City: Australian Sppech Science and Technology Association.

Karttunen, Lauri. 1974. Presupposition and linguistic context. Theoretical Linguistics 1(1-3). 181-194. doi:10.1515/thli.1974.1.1-3.181.

Lee, Chungmin. 2018. Non-factive alternants of the attitude verb 'know' in Korean, Turkish, and Hungarian. Journal of The National Academy of Sciences. Republic of Korea 58(1). 37-85.

Mahler, Taylor, Marie-Catherine de Marneffe \& Catherine Lai. 2019. The prosody of presupposition projection in naturally-occuring utterances. Poster presented at Sinn und Bedeutung $(\mathrm{SuB}) 24$.

Moulton, Keir. 2009. Natural Selection and the Syntax of Clausal Complementation: UMass Amherst PhD dissertation.

Nairn, Rowan, Cleo Condoravdi \& Lauri Karttunen. 2006. Computing relative polarity for textual inference. In The 5th International Workshop on Inference in Computational Semantics (ICoS 5), 20-29. Buxton, England: Association for Computational Linguistics.

Özyıldız, Deniz. 2017a. Attitude reports with and without true belief. Semantics and Linguistic Theory (SALT) 27. 397-417. doi:10.3765/salt.v27i0.4189.

Özy1ld1z, Deniz. 2017b. Factivity and prosody in Turkish attitude reports. Generals paper, UMass Amherst.

Özyıldız, Deniz. 2018. A prosodic factivity alternation explained by clause movement. Paper presented at Semantics and Linguistic Theory (SALT) 28.

Roberts, Craige. 1996. Information structure in discourse: Towards an integrated formal theory of pragmatics. OSU Working Papers in Linguistics 49.

Rochemont, Michael. 2016. Givenness. In Caroline Fry \& Shinichiro Ishihara (eds.), The Oxford Handbook of Information Structure, Oxford: Oxford University Press.

Roelofsen, Floris \& Wataru Uegaki. 2020. Searching for a universal constraint on the possible denotations of clause-embedding predicates. Semantics and Linguistic Theory (SALT) 30. To appear.

Romoli, Jacopo. 2014. The presuppositions of soft triggers are obligatory scalar implicatures. Journal of Semantics 32(2). 173-219. doi:10.1093/jos/fft017.

Rooth, Mats. 1992. A theory of focus interpretation. Natural Language Semantics 1(1). 75-116. doi:10.1007/bf02342617.

van der Sandt, Rob A. 1992. Presupposition projection as anaphora resolution. Journal of Semantics 9(4). 333-377. doi:10.1093/jos/9.4.333.

Schwarzschild, Roger. 1999. GIVENness, AvoidF and other constraints on the placement of accent. Natural Language Semantics 7(2). 141-177. doi:10.1023/A:1008370902407. 
Prosodically-conditioned factive inferences

Simons, Mandy, David Beaver, Craige Roberts \& Judith Tonhauser. 2017. The best question: Explaining the projection behavior of factives. Discourse Processes 54(3). 187-206. doi:10.1080/0163853x.2016.1150660.

Spector, Benjamin \& Paul Egré. 2015. A uniform semantics for embedded interrogatives: An answer, not necessarily the answer. Synthese 192(6). 1729-1784. doi:10.1007/s11229-015-0722-4.

Stalnaker, Robert. 1977. Pragmatic presuppositions. In Semantics and Philosophy, 135-148. New York: New York University.

Steinert-Threlkeld, Shane. 2020. An explanation of the veridical uniformity universal. Journal of Semantics 37(1). 129-144. doi:10.1093/jos/ffz019.

Theiler, Nadine, Floris Roelofsen \& Maria Aloni. 2018. A uniform semantics for declarative and interrogative complements. Journal of Semantics 35(3). 409-466. doi:10.1093/jos/ffy003.

Tonhauser, Judith. 2016. Prosodic cues to presupposition projection. Semantics and Linguistic Theory (SALT) 26. 934-960. doi:10.3765/salt.v26i0.3788.

Tonhauser, Judith, David I Beaver \& Judith Degen. 2018. How projective is projective content? gradience in projectivity and at-issueness. Journal of Semantics 35(3). 495-542. doi:10.1093/jos/ffy007.

Wagner, Michael. 2012. Focus and givenness: A unified approach. In Ivona Kucerova \& Ad Neeleman (eds.), Contrasts and positions in information structure, 102-147. Cambridge, UK: Cambridge University Press. doi:10.1017/cbo9780511740084.007.

White, Aaron Steven. 2019. Lexically triggered veridicality inferences. In J. Östman \& J. Verschueren (eds.), Handbook of Pragmatics, Amsterdam: John Benjamins Publishing Company.

White, Aaron Steven \& Kyle Rawlins. 2018. The role of veridicality and factivity in clause selection. In The 48th annual meeting of the North East Linguistic Society (NELS 48), Amherst, MA: GLSA Publications.

Sunwoo Jeong

Department of Linguistics

Gwanak-gu, Gwanak-ro 1, 2-108

Seoul National University

sunwooj@snu.ac.kr 